\title{
ANTOFAGASTA: APUNTES PARA UNA BIOCIUDADANÍA CONTRA LA NECROPOLÍTICA ECOTOXICOLÓGICA*
}

\author{
ANTOFAGASTA: NOTES FOR A BIOCITIZENSHIP AGAINST \\ ECOTOXICOLOGICAL NECROPOLITICS
}

\author{
Augusto Obando Cid ${ }^{1}$ \& Gonzalo Infante Grandón ${ }^{2}$ \\ augusto.obando@ufrontera.cl - gonzalo.infante@ufrontera.cl \\ Universidad de La Frontera \\ Temuco, Chile \\ DOI: https://doi.org/10.32735/S2735-61752020000117144
}

\section{RESUMEN}

El presente texto es una revisión de antecedentes ecotoxicológicos, respecto de la contaminación del norte chileno y, particularmente, acerca de la contaminación por metales pesados y sustancias inorgánicas en Antofagasta; para reflexionar en torno al concepto de Necropolítica (Mbembe, 2011) y Necrocapitalismo (Banerjee, 2008).

Considerando la evidencia empírica de la contaminación generada por la Gran Minería, en la Región de Antofagasta, esta debe ser entendida como acción Necropolítica; ya que la generación de riqueza minera, debido al proceso productivo, genera contaminación, lo que deteriora la salud y -dada su característica dosis/dependiente- es un proceso de producción de muerte. Se plantea que el costo vital de la producción económica capitalista minera, es a expensas de vidas humanas, que se sacrifican como contrapartida de este Necrocapitalismo.

Por último, se releva la importancia fundamental del accionar individual/colectivo de la comunidad afectada, planteándose la aparición de un sujeto movimental; que fundamenta su acción colectiva en su propia condición biológica, como sujeto enfermo/contaminado (Petryna, 2002) por metales pesados y compuestos inorgánicos. En tanto, a través de la abogacía y accountability, emplazan al gobierno y sector empresarial de la minería, exigiendo acciones concretas, en relación con el derecho a la protección de la salud individual y colectiva.

Palabras clave: Biociudadanía; Ecotoxicología; Necropolítica; Necrocapitalismo.

\section{ABSTRACT}

The present text is an ecotoxicological background review, regarding contamination in northern Chile and, in particular, about contamination by heavy metals and inorganic substances in Antofagasta; to reflect on the concept of Necropolitics (Mbembe, 2011) and Necrocapitalism (Banerjee, 2008). Considering the empirical evidence of pollution generated by large mining, in the Antofagasta Region, it must be understood as Necropolitical action; because the generation of mining wealth, due to the production process, generates pollution, which deteriorates health

\footnotetext{
* Artículo recibido el 1 de junio de 2019; aceptado el 30 de junio de 2019.

1 Doctor en Ciencias Sociales. Adscrito al Departamento de Salud Pública, Facultad de Medicina, Universidad de La Frontera.

${ }^{2}$ Doctorando en Salud Colectiva. Adscrito al Departamento de Salud Pública, Facultad de Medicina, Universidad de La Frontera.
} 
and -given its dose/dependent characteristic- is a process of production of death. It is proposed that the vital cost of capitalist mining economic production is at the expense of human lives, which are sacrificed in return for this Necrocapitalism. Finally, the fundamental importance of individual/collective action of the affected community is relieved, with the emergence of a moving subject being raised; which bases its collective action on its own biological condition, as a sick/contaminated subject (Petryna, 2002) by heavy metals and inorganic compounds. Meanwhile, through advocacy and accountability, they place the government and business sector of mining, demanding concrete actions, in relation to the right to protection of individual and collective

health.

Keywords: Biocitizen; Ecotoxicology; Necropolitics; Necrocapitalism.

\section{Introducción}

\section{Antofagasta y el legado del salitre: del extractivismo desarrollista a la contaminación de la Gran Minería}

El norte de Chile y, en específico, la Región de Antofagasta, presentan como principal actividad económica la minería, sin embargo, dicha actividad no está exenta de la problemática medioambiental que genera. La explotación del salitre es el prolegómeno extractivista, que configuró lo que es hoy la contaminación por metales pesados en la ciudad de Antofagasta.

La red de ferrocarriles y el inicio de la construcción del puerto en Antofagasta en 1913 inaugurado en 1943 por el presidente Juan Antonio Ríos-, con más de 30.000 metros ganados al mar, son los hitos históricos de la conformación de una infraestructura portuaria; acorde con las necesidades del modelo extractivista desarrollista, basado en la explotación de recursos naturales, siendo los productos de la minería los principales de esta región.

Desde la industrialización del proceso extractivo de la Gran Minería (GM), en la Región de Antofagasta, se ha generado progresivamente contaminación ambiental, la cual ha afectado a diversas localidades, pero en especial a la ciudad de Antofagasta; debido al puerto presente en esta, lugar en el que se realiza la exportación del producto final de la extracción minera.

Recién en la última década, la contaminación por sustancias como el arsénico, plomo y otros metales pesados, ha comenzado a tener importancia; debido a la medición de dicha contaminación y a la producción de evidencia científica a nivel mundial, acerca de los niveles de contaminación considerados peligrosos para la vida humana; determinándose que los altos niveles de concentración de contaminantes (fundamentalmente metales pesados y sustancias inorgánicas), comprometen gravemente la salud, generando procesos patológicos asociados a la alta exposición de estos contaminantes, lo que puede generar elevadas tasas de letalidad y morbi-mortalidad en la población.

Sin embargo, la contaminación ambiental y humana, generada por el proceso extractivista de la GM, tardó mas de 40 años en ser foco de atención; recién en el año 1989 aparecieron las primeras denuncias de la población, frente al mal manejo del acopio de material minero en el puerto. Cuatro años más tarde, en el año 1993, se realizaron los primeros estudios epidemiológicos y medioambientales. Según Hernando: 
"Las denuncias de la comunidad comenzaron en 1989. Posteriormente, en 1992, el Servicio de Salud de Antofagasta otorgó el plazo de un año al FCAB para el traslado de los minerales fuera del radio urbano. Dado que no hubo respuesta a esa exigencia, en 1993 la Cancillería protestó, pues se trataba de materiales que estaban protegidos por el Tratado de 1904. Ese mismo año el Servicio de Salud realizó un estudio epidemiológico en escolares de los sectores afectados, vale decir, en las escuelas del entorno; sin embargo, sus resultados fueron refutados por la Cancillería, aduciendo que no había contramuestras, ni grupo de control y otras debilidades técnicas" (Hernando en Cámara de Diputados, 2016, p. 39).

Lo anterior da cuenta de la contaminación por metales pesados, que posteriormente derivó en un estudio sobre la contaminación plúmbica, que será la principal preocupación en esta época (Eterovic, 1998), (Sepúlveda A, Vega M, \& Delgado B, 2000).

En el año 2015, más de 25 años después de las anteriores denuncias e investigaciones sobre la contaminación en Antofagasta, se constituye en la cámara de Diputados, la Comisión Especial Investigadora (CEI) de la actuación de los organismos públicos, encargados de la protección de la salud y el medio ambiente, en la comuna de Antofagasta. La CEI realizó un informe de cuatrocientas páginas, describiendo la problemática de la contaminación; planteando la necesidad de la revisión de la Ley 19.300, Ley sobre Bases Generales del Medio Ambiente, indicaciones que no se han efectuado (Cámara de Diputados, 2016).

Es importante referir al hecho de que, en el documento, se puntualiza que la contaminación percibida por la población es plúmbica, sin embargo, se plantea una contaminación sistemática por varios otros metales pesados y sustancias inorgánicas; circunstancias que, al parecer, la población y las autoridades desconocen, ya que, respecto de los efectos agudos y crónicos, frente a la exposición dosis-dependiente de dichos agentes contaminantes, no se hace referencia en el documento.

Por otra parte, la Cámara de Diputados ha oficiado otras comisiones de investigación, por situaciones similares de contaminación; como la producida en Concón, Quintero y Puchuncaví (Cámara de Diputados, 2019), las cuales cobraron notoriedad por el movimiento social que generaron.

El presente texto, por tanto, da cuenta de una tensión, entre las diversas fuentes de evidencia acerca de la contaminación generada por la GM en Chile, en la Región de Antofagasta, y su lectura como acción Necropolítica. Lo que no solo genera un deterioro de la salud, sino que también debe ser entendido como un proceso de producción de muerte, sustentado en la generación de riqueza de la industria minera; en otros términos, sería el costo vital y de vidas humanas, que se pueden sacrificar, como parte del importe de la producción de la economía minera. 


\section{Material y método}

El presente estudio se plantea como una investigación documental, sustentado en la realización de una revisión bibliográfica sistemática de literatura científica -en el campo de la biomedicina-, en relación con la evidencia actual sobre contaminación por metales pesados y sustancias inorgánicas, según parámetros epidemiológicos y de salud ambiental; para ello se revisaron las bases de datos de bibliografía científica: Medline, LILACS, PubMed, Biblioteca Cochrane Plus y Web of Science.

Análogamente, se realizó una estrategia de búsqueda complementaria en fuentes secundarias, en lo referente a mediciones realizadas por el Instituto de Salud Publica (ISP), las denuncias del Colegio Médico de Chile y las actas de la CEI contenidas en la Biblioteca del Congreso Nacional $(\mathrm{BCN})$, referidas a la contaminación en la ciudad de Antofagasta, con relación a metales pesados y sustancias inorgánicas; para compararlos con los parámetros de contaminación actuales.

Por último, se realizó un análisis teórico de dichos antecedentes, en virtud de los conceptos de Biociudadanía propuestos por Rose (2004) y Petryna (2002); Necropolítica de Mbembe (2011) y Necrocapitalismo de Banerjee (2008); para interpretar las acciones movimentales de las personas afectadas en Antofagasta.

\section{Antofagasta: lugar de un ensayo clínico a escala regional}

\section{La evidencia de contaminación por arsénico}

El arsénico es un elemento que está presente, de forma natural, en la corteza terrestre; destacando entre sus características que, en su forma inorgánica, es muy tóxico. El principal problema de salud pública que provoca es la contaminación del agua, ya que -someterse a su exposición prolongada- genera daños agudos y crónicos (Organización Mundial de la Salud [OMS], 2004).

El Dr. Aliro Bolados (2015), señala que Chile y la Región de Antofagasta poseen características especiales, para estudiar los efectos a gran escala de la contaminación por metales pesados y en especial la contaminación por arsénico; la primera de ellas es que dispone de buenos registros, la segunda versa en que existe una dosis de exposición bien reportada y la tercera consiste en que, dicha exposición, tiene un tiempo delimitado. Por lo tanto, la Región de Antofagasta, puede considerarse como un Ensayo Clínico a escala regional (Fraser, 2012).

La variable, de dicho estudio, es la exposición al arsénico; la cual ha sido manejada a priori y con antelación, dado que la exposición continua, de alguna u otra forma, ha permitido evidenciar sus efectos a posterior. Con ello, el hallazgo de cáncer, en la Región de Antofagasta, responde a las lógicas de un Ensayo Clínico, según lo plantea Fraser:

En Antofagasta, la exposición fue severa, pero de duración limitada. Hasta mediados del siglo XX, la región, escasamente poblada, extrajo agua potable de un río libre de arsénico. Cuando la población surgió, con un auge minero, en la década de 1950, el sistema de agua era ampliado para incluir otros dos ríos. Aumentaron las 
concentraciones de arsénico diez veces y los residentes fueron expuestos a niveles tanto como 17 veces mayores a la recomendación de la OMS (Fraser, 2012: 379) (Traducido para el presente texto).

Lo recién planteado, se debió especialmente a la configuración del modelo capitalista extractivo de materias primas, cuya principal actividad -hasta el día de hoy- es la GM del cobre. Su mayor apogeo inició con la explotación por parte del Estado, a mediados del siglo pasado; luego, debido al modelo de desarrollo y económico, se incluyó la participación de privados. Cabe destacar que estos últimos, dadas las estructuras legislativas, obtuvieron altos beneficios económicos, con nula previsión del daño ambiental y a la salud que provocaba dicha actividad, considerada inocua hasta fines del siglo pasado.

En el periodo histórico inicial del extractivismo, se plantea que la extracción minera no tiene relación con la contaminación y sus características especificas en la Región de Antofagasta. Por ello, la presencia de arsénico en las ciudades del norte de Chile, no es un "efecto" de la actividad minera, sino de su carácter volcánico (Fraser, 2012).

Sin embargo, el discurso de la "inocuidad" - de dichas acciones de extracción- comienza a cambiar, basándose en la evidencia científica; con ello, se plantea que la producción minera acarrea contaminación ambiental, la cual -indefectiblemente- afectará a la población humana.

Un claro ejemplo de esta transformación discursiva, de una sustancia inocua a una nociva, gira en torno a la contaminación por arsénico; dado que, respecto del desierto chileno, se afirma que, por razones naturales y propias, posee altas concentraciones de dicho elemento (ver tablas 1 y 2 ).

\section{Tabla 1}

Clasificación de riesgo del Arsénico, según el International Agency for Research on Cancer (IARC).

\begin{tabular}{|l|l|l|}
\hline $\begin{array}{l}\text { Año } \\
1973\end{array}$ & IARC & $\begin{array}{l}\text { Efecto } \\
\text { Vol. 2 }\end{array}$ \\
$\begin{array}{l}\text { Clasificación grupo 3, } \\
\text { No puede ser clasificado como carcinogénico en humanos. }\end{array}$ \\
\hline 1980 & Vol. 23 & $\begin{array}{l}\text { Clasificación grupo 1, } \\
\text { Evidencia suficiente para cáncer de piel y pulmón. }\end{array}$ \\
\hline 2004 & Vol. 84 & $\begin{array}{l}\text { Clasificación grupo 1, } \\
\text { Evidencia suficiente para cáncer de piel, pulmón y vejiga. }\end{array}$ \\
\hline
\end{tabular}

Fuente: IARC, en Bolados (2015).

En la tabla 1, es posible observar la transformación de un compuesto inocuo a cancerígeno, según el nivel de evidencia disponible y las investigaciones que permiten corroborarlo. Por lo tanto, cuando una sustancia se considere inofensiva para la salud, pero se sospeche de su potencial peligrosidad, será necesario realizar estudios concernientes a refutar dicha hipótesis de inocuidad. En la actualidad, diversos son los estudios que dan cuenta de los efectos de la contaminación por metales pesados, entre ellos el plomo y el arsénico (presentes en la población de Antofagasta), lo que se problematiza en el presente texto. 


\section{Tabla 2}

Niveles de toxicidad del Arsénico (As) en agua potable (mcg- $\mu g / /)^{3}$ según la OMS (1958-2006).

\begin{tabular}{|l|l|l|l|}
\hline Organismo & Año & $\begin{array}{c}\text { Nivel considerado } \\
\text { tóxico }\end{array}$ & \multicolumn{1}{|c|}{ Descripción de la evidencia } \\
\hline OMS & 1958 & 200 & As: tóxico para salud humana. \\
\hline OMS & 1963 & 50 & As: tóxico para salud humana. \\
\hline OMS & 1971 & 50 & $\begin{array}{l}\text { As: ¿carcinogénico humano? sin } \\
\text { pruebas establecidas. }\end{array}$ \\
\hline OMS & 1984 & 50 & $\begin{array}{l}\text { As: componentes inorgánicos } \\
\text { significativos para la salud. }\end{array}$ \\
\hline OMS & 1993 & 10 & As: cáncer a la piel en Taiwán. \\
\hline OMS & 2006 & $>0,01$ & As: tóxico para salud humana. \\
\hline
\end{tabular}

Fuente: Elaboración propia en base a Bolados (2015).

La tabla 2 delimita las normas actuales, respecto de la potencialidad toxica del arsénico, a bajas concentraciones en el agua potable. A nivel internacional, el caso de Chile es seguido con atención, debido a las implicancias para determinar causalidad en el efecto; como se ha realizado en Taiwán y Bangladesh, según investigaciones de Smith et al. (2002), Harlt et al. (2004), Ravencrof (2007), Bagchi (2007) y Steinmaus et al. (2013).

La importancia de lo anterior radica en que cada país acoge las normativas de contaminación que considere más relevantes. Sin embargo, estas normativas cambian según los hallazgos científicos, por lo tanto, el nivel de contaminación que en los años 90 era considerado inocuo, puede -al cabo de algunos años- llegar a ser considerado de alta toxicidad para la población humana. En lo referente a la contaminación por arsénico, comienza -incluso- antes de plantearse como problema de Salud Pública a nivel mundial y nacional.

\footnotetext{
${ }^{3}$ Se refiere a Microgramos por Litros, un microgramo equivale a la milmillonésima parte de un kilogramo (10

${ }^{9} \mathrm{~kg}$ ) o a la millonésima parte de un gramo $\left(10^{-6} \mathrm{~g}\right)$.
} 


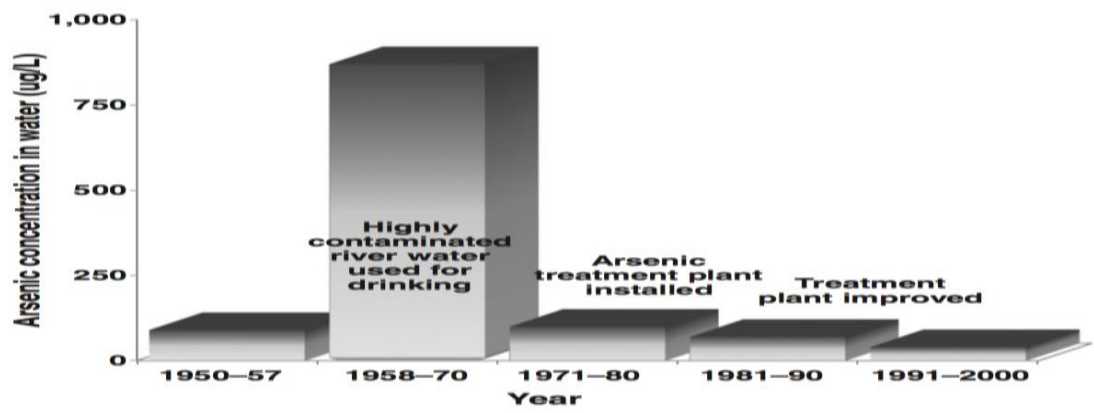

Figura 1. Gráfico 1. Concentración de Arsénico (As) en el agua para consumo (Antofagasta, Chile). Fuente: Steinmaus et al (2013).

La premisa de traslación inocuidad-toxicidad, en el caso del arsénico, se observa claramente en el gráfico 1. La contaminación, por dicho compuesto, inició en la década de los 50' (según los registros), generándose una suerte de contaminación masiva, de casi $860 \mathrm{mcg}-\mu \mathrm{g} / \mathrm{l}$, en el agua de consumo humano, desde 1958 a 1970, según lo plantean Fraser (2012) y Steinmaus et al. (2013). Posteriormente, se logró reducir la exposición -solo en forma parcial-, mediante las plantas de tratamiento de aguas. Sin embargo, Fraser (2012) plantea que las exposiciones fueron severas, generándose una suerte de clúster (ensayo clínico); produciéndose una serie de daños a la salud, como enfermedades cardiovasculares, diabetes, bronquiectasias y enfermedad pulmonar obstructiva crónica (EPOC), efectos reproductivos y varios tipos de cánceres (pulmón, piel y vejiga) (Smith et al, 2009), (Steinmaus et al, 2013).

\section{Tabla 3}

Concentración histórica de Arsénico (As) en agua potable según área y año.

Average arsenic concentration, $\mu \mathrm{g} / \mathrm{L}$

\begin{tabular}{|c|c|c|c|c|c|c|c|c|c|}
\hline \multirow[b]{3}{*}{ Region } & \multirow[b]{3}{*}{ City or town } & \multirow[b]{3}{*}{ Population $^{a}$} & & & & & & & \\
\hline & & & \multicolumn{7}{|c|}{ Years } \\
\hline & & & 1930-1957 & 1958-1970 & 1971-1977 & 1978-1979 & 1980-1987 & 1988-1994 & $1995+$ \\
\hline \multirow[t]{6}{*}{1} & Arica & 168,594 & 10 & 10 & 10 & 10 & 10 & 10 & 9 \\
\hline & Putre & 1,799 & 1 & 1 & 1 & 1 & 1 & 1 & 1 \\
\hline & Iquique & 196,941 & 60 & 60 & 60 & 60 & 60 & 60 & 10 \\
\hline & Huara & 2,365 & 30 & 30 & 30 & 30 & 30 & 30 & 30 \\
\hline & Pica & 5,622 & 10 & 10 & 10 & 10 & 10 & 10 & 10 \\
\hline & Pozo Almonte & 9,855 & 40 & 40 & 40 & 40 & 40 & 40 & 40 \\
\hline \multirow[t]{8}{*}{$\|$} & Tocopilla & 21,827 & 250 & 250 & 636 & 110 & 110 & 40 & 10 \\
\hline & Maria Elena & 6,852 & 250 & 250 & 636 & 110 & 110 & 39 & 39 \\
\hline & Calama & 125,946 & 150 & 150 & 287 & 110 & 110 & 40 & 38 \\
\hline & San Pedro & 4,522 & 600 & 600 & 600 & 600 & 600 & 600 & 600 \\
\hline & Antofagasta & 270,184 & 90 & 860 & 110 & 110 & 70 & 40 & 10 \\
\hline & Mejillones & 7,660 & 90 & 860 & 110 & 110 & 70 & 37 & 10 \\
\hline & Taltal & 10,101 & 60 & 60 & 60 & 60 & 60 & 60 & 60 \\
\hline & Recent migrants & 82,312 & $<10$ & $<10$ & $<10$ & $<10$ & $<10$ & $<10$ & $<10$ \\
\hline
\end{tabular}

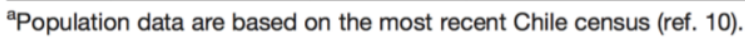

Fuente: Steinmaus et al (2013). 
La tabla 3, muestra los niveles de exposición al arsénico, en población de la Región de Antofagasta. En ella se observa, claramente, que la problemática de la contaminación, no está circunscrita solo al lugar crítico denunciado -inicialmente- por el Colegio Médico y la Diputada Hernando (Camara de Diputados, 2016); Tocopilla, María Elena y la ciudad de Antofagasta, fueron contaminadas con arsénico de forma masiva, hasta la década de los 90'. Sin embargo, la localidad de San Pedro mantiene niveles de contaminación alarmantes, de $600 \mathrm{mcg}-\mu \mathrm{g} / \mathrm{l}$, hasta la época actual.

Steinmaus et al $(2007,2013)$ plantean que las personas que nacieron entre los años 1930 y 1950, fueron afectadas por arsénico de forma aguda, en el período reproductivo, presentando mortalidad asociada a enfermedades cardiovasculares (ver gráfico 2). Por otro lado, las personas que nacieron desde el año 1958 al año 1970, se enfrentaron a una intoxicación aguda de arsénico (intrauterina y de la primera infancia), que les causaría mortalidad por cáncer, observable desde el año 2000 y en adelante (ver gráfico 3).

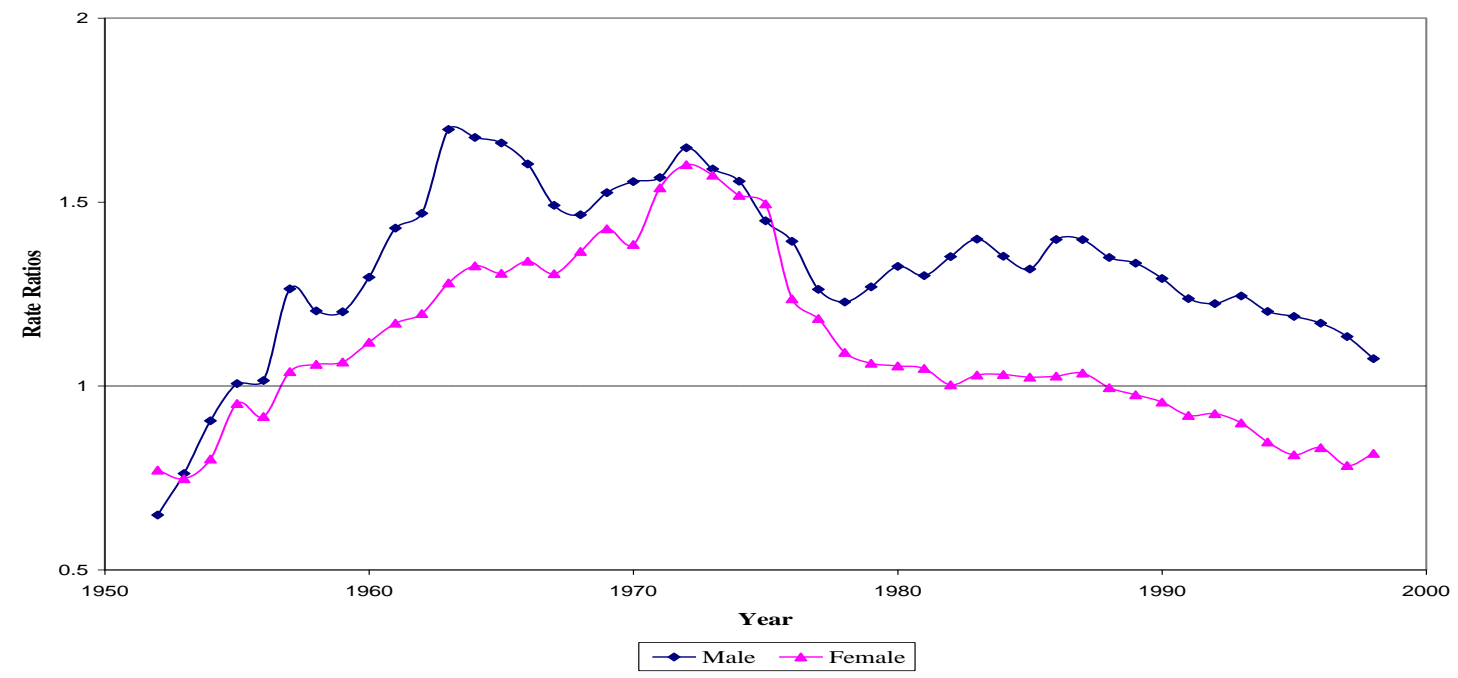

Figura 2. Gráfico 2. Mortalidad por Infarto Agudo al Miocardio (IAM) en relación con la alta exposición a Arsénico (As) (Antofagasta, Chile). Fuente: Steinmaus et Al $(2007,2013)$.

El gráfico describe la mortalidad por IAM, enfermedad cardiovascular que se asocia a la exposición por arsénico, según Steinmaus (2007, 2013). Cabe destacar que, en el período 1960-1970, el perfil epidemiológico chileno presentaba como principales causas de muerte las enfermedades infecto-contagiosas, con mínima incidencia de patologías de tipo crónicas no transmisibles; lo que da cuenta de una alteración del perfil epidemiológico en la población de Antofagasta, relacionado con la exposición al arsénico. 


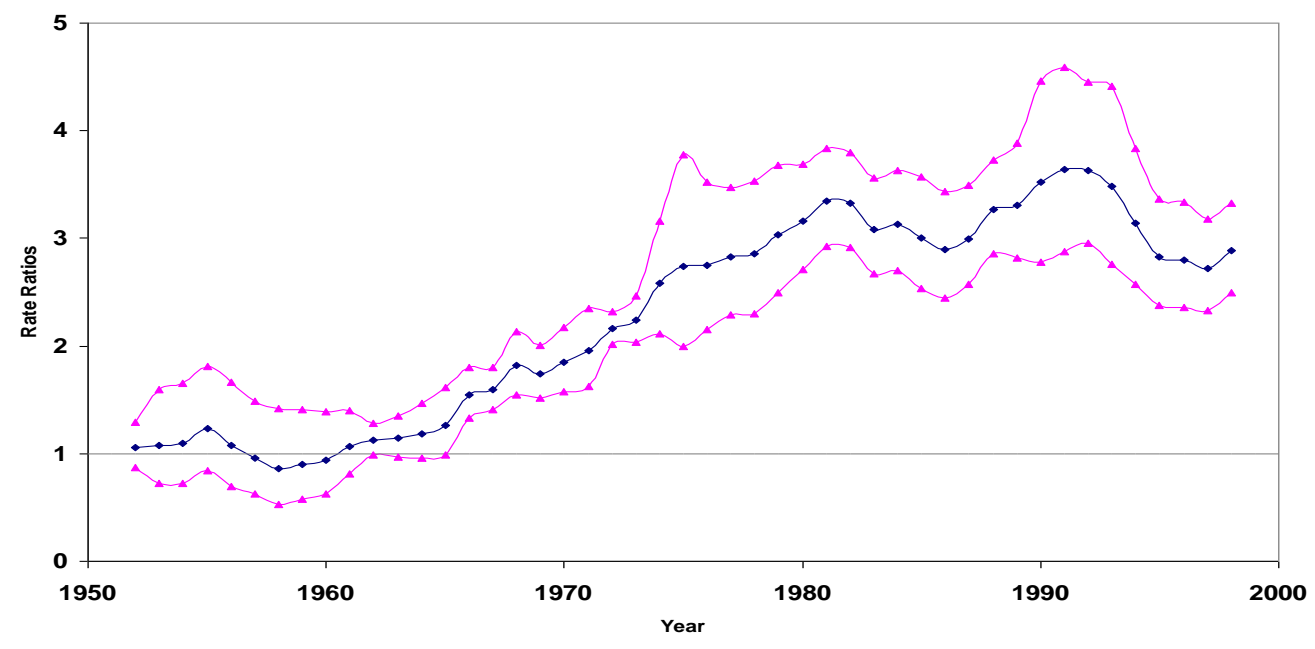

$\rightarrow$ Rate ratios $\leadsto$ Lower $95 \% \mathrm{Cl} \leadsto$ Upper $95 \% \mathrm{Cl}$

Figura 3. Gráfico 3. Latencia de la Mortalidad por Cáncer de Pulmón (Antofagasta, Chile). Fuente: Steinmaus et Al (2007, 2013).

El gráfico 3 presenta la mortalidad por cáncer de pulmón, asociada a la contaminación por arsénico. En el caso chileno, la mortalidad por cáncer es producida -principalmente-por los de cánceres de vesícula y estómago; que tienen un aumento posterior a 1990, con la transformación del perfil epidemiológico. En Antofagasta, la mortalidad por cáncer comienza, prematuramente, a mediados de la década del 70', mostrando un incremento, ostensible, en la década de los 90'. Por lo tanto, se puede inferir que las personas que sobrevivieron a la mortalidad por IAM, se ven enfrentadas, posteriormente, a la mortalidad por cáncer de pulmón u otras patologías oncológicas, como cáncer de vejiga (Marshall et al, 2007) o afecciones pulmonares (Smith et al, 2011), ( Steinmaus et al, 2013).

El Departamento de Estadística e Información en Salud (DEIS) y sus Reportes de Estadísticas Vitales, desde los años 70', dan cuenta de un aumento progresivo en la mortalidad por cánceres de piel, pulmón, próstata, mama y estómago en la Región de Antofagasta (DEIS, 2003-2007).

\section{Antofagasta: del puerto a la ciudad}

\section{Contaminación por metales pesados}

Según Hernando (2015), existen diversos registros -desde la década de los 90'-, para delimitar la problemática de la contaminación por plomo (intoxicación plúmbica). A través de estudios transversales en los años 1993 y 1998; monitoreo de la calidad del aire, desde el año 1991 al año 1998; estudio en población escolar, en el año 1998; muestreo de plomo en suelo, polvo, sedimentos y agua, en el año 1998. Registros que permitieron determinar que la contaminación plúmbica, afectaba al $47 \%$ de la población de Antofagasta.

En consecuencia, se implementaron medidas paliativas y de contención para dicha contaminación; medidas que, según Hernando (2015), fueron exitosas, de acuerdo con los estudios realizados por la Comisión Nacional del Medio Ambiente (CONAMA) y la Secretaría 
Regional Ministerial de Salud (SEREMI SALUD) de Antofagasta, en el año 2006. Los hallazgos indicaron que ya no existiría riesgo de exposición a plomo, destacando que el sector de la "ruta de camiones con concentrado de plomo y zinc", en el tramo urbano, presentaba excedencias a la norma (sobre $300 \mathrm{mg} / \mathrm{kg}$ ), en el $80 \%$ de las muestras tomadas.

Sin embargo, frente a dichas mediciones, el Colegio Médico de Chile y Regional de Antofagasta, denunciaron que se mantiene la alta contaminación, refutando e impugnando las mediciones previas, exigiendo una investigación al ISP4; la que fue realizada el año 2014, en el borde costero de toda la ciudad. Aun cuando la Norma holandesa utilizada, plantea que el límite máximo de concentración de plomo $(\mathrm{Pb})$ es de $600 \mathrm{mg} / \mathrm{kg}$, para considerarlo tóxico para la salud, este es superado en muchos lugares del puerto.

Lo significativo de la medición realizada por el ISP, es la medición del Arsénico (As); medición que, de acuerdo con la norma anterior, fija el límite de concentración, para ser considerado tóxico, en $50 \mathrm{mg} / \mathrm{kg}$, límite superado con creces en dicha medición. Al parecer, las medidas solo estuvieron abocadas a realizar acciones paliativas frente a la contaminación plúmbica; lo que no impidió, a través de las mediciones, la visibilización de otros componentes de mayor toxicidad para el ser humano (ver figura 4).

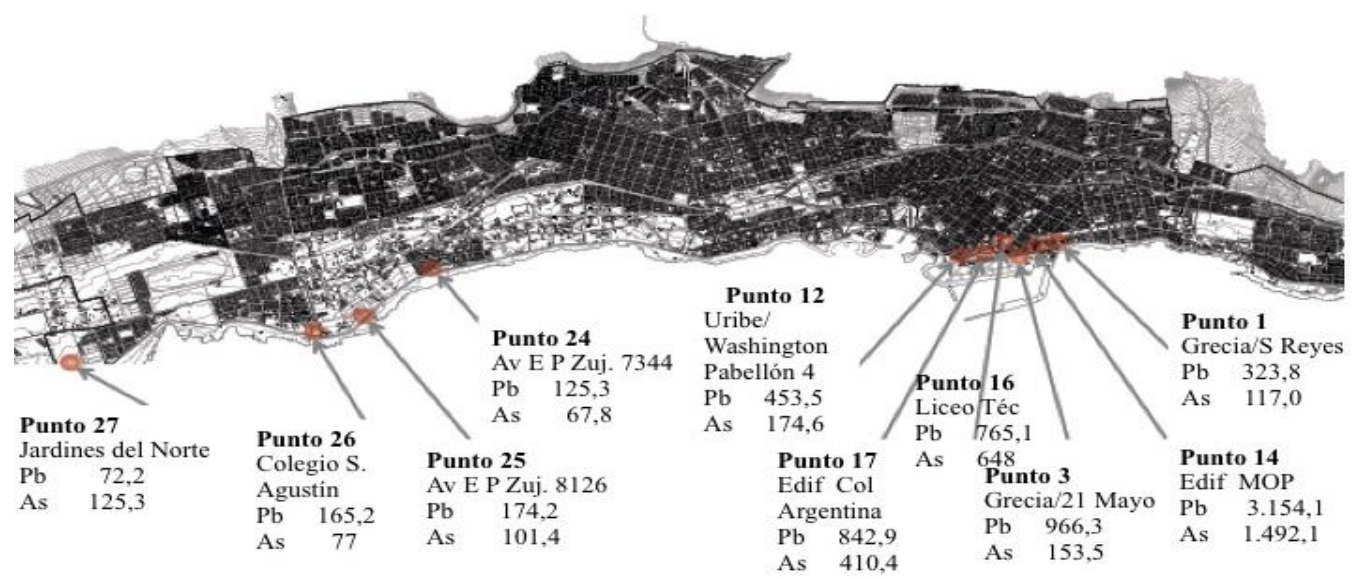

Figura 4. Estudio ISP 2014 Borde Costero, ciudad de Antofagasta. Metales Pesados en polvo sedimentable medidos en mg/kg (Arsénico [As] y Plomo [Pb]). Fuente: ISP (2014) en Hernando (2015).

En la figura 4, en relación con el arsénico, observamos que lo descrito, en el apartado anterior, es mucho más complejo que solo la contaminación de las aguas; se puede apreciar la difícil situación que enfrenta la ciudad, debido a que, por un lado, está expuesta por el consumo de agua potable y, por otro, de forma superpuesta, se enfrenta a la exposición por suspensión en su forma inorgánica, la que destaca por ser más tóxica.

Por otro lado, en el mismo estudio, se realizaron mediciones de otros metales pesados, arrojando una problemática más compleja que solo la contaminación plúmbica, develando una

$4 \quad$ Ver: http://radio.uchile.cl/2014/10/31/isp-confirma-de-presencia-de-arsenico-y-plomo-en-el-suelo-deantofagasta. 
suerte de exposición sistemática, a un sinnúmero de compuestos tóxicos para la salud de la población (ver tabla 4 y 5).

\section{Tabla 4}

Normativa holandesa para suelos contaminados con Metales Pesados y Compuestos Inorgánicos.
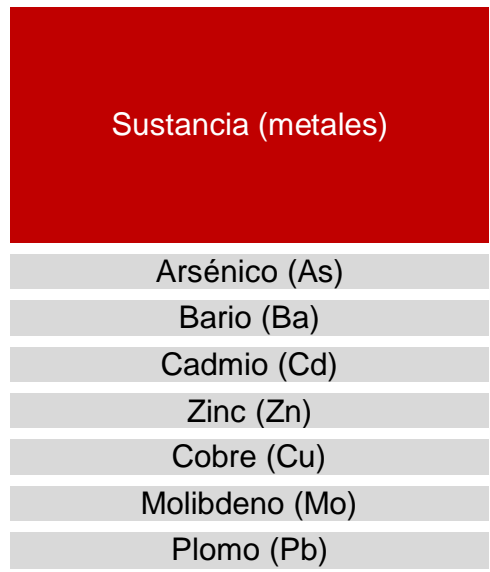

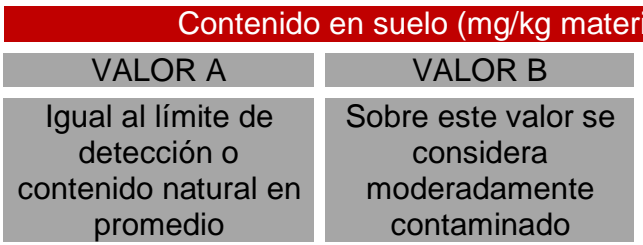

$$
0,1
$$$$
200
$$

$$
0
$$$$
0,5
$$$$
0,1
$$$$
10
$$

0,1

$$
30
$$$$
400
$$

5

500

100

40

150
VALOR C

Sobre este valor se considera contaminado

50

2000

20

3000

500

200

600

Fuente: ISP (2014) en Hernando (2015), Bolados (2015). 
Tabla 5

Metales Pesados y Compuestos Orgánicos en la ciudad de Antofagasta, según Normativa holandesa.

\begin{tabular}{|c|c|c|c|c|c|c|c|c|c|c|}
\hline \multirow{13}{*}{$\begin{array}{l}\text { Sustancia } \\
\text { (metales) }\end{array}$} & \multirow{11}{*}{$\begin{array}{l}\text { Grecia/ } \\
\text { Salvado } \\
\text { r Reyes }\end{array}$} & \multirow{11}{*}{$\begin{array}{c}\text { Greci } \\
\text { a/21 } \\
\text { de } \\
\text { mayo }\end{array}$} & \multirow{11}{*}{$\begin{array}{c}\text { Uribe/ } \\
\text { Balmac } \\
\text { eda }\end{array}$} & \multirow{11}{*}{$\begin{array}{l}\text { Edificio } \\
\text { MOP, } \\
\text { Frente } \\
\text { al } \\
\text { Puerto } \\
\text { Cara } \\
\text { Interior }\end{array}$} & \multirow{11}{*}{$\begin{array}{l}\text { Frontis } \\
\text { Liceo } \\
\text { Técnico }\end{array}$} & \multicolumn{4}{|c|}{ Av. } & \multirow{11}{*}{$\begin{array}{c}\text { Condominio } \\
\text { Jardines de } \\
\text { Norte VI }\end{array}$} \\
\hline & & & & & & & Av. & Edmund & & \\
\hline & & & & & & & Edmund & o Pérez & & \\
\hline & & & & & & Edificio & o Pérez & Zujovic & & \\
\hline & & & & & & Colecti & Zujovic & Entre & Colegio & \\
\hline & & & & & & vo & 7344 & Parque & San & \\
\hline & & & & & & Argenti & Altura & Nicolás & Agustín & \\
\hline & & & & & & na & Parque & Tirado y & & \\
\hline & & & & & & & Los & playa el & & \\
\hline & & & & & & & Pinares & Trocader & & \\
\hline & & & & & & & & 0 & & \\
\hline & Punto & Punto & Punto & Punto & Punto & Punto & Punto & Punto & Punto & Punto \\
\hline & 1 & 3 & 12 & 14 & 16 & 17 & 24 & 25 & 26 & 27 \\
\hline Arsénico (As) & 117,0 & 153,5 & 164,0 & 1492,1 & 648,0 & 410,4 & 67,8 & 101,4 & 77 & 72,2 \\
\hline Bario (Ba) & 33,2 & 55,7 & 29,5 & 47,5 & 268,5 & 145,7 & 224,5 & 205,4 & 510,1 & 328,9 \\
\hline Cadmio (Cd) & 28,2 & 78,2 & 21,7 & 128,7 & 43,4 & 48,1 & 7 & 6 & 5,5 & 4,3 \\
\hline Zinc (Zn) & 6500,7 & 17614 & 15867,7 & 30545,7 & 9700,8 & $\begin{array}{c}10229 \\
8\end{array}$ & 2102 & 3149,3 & 1646,9 & 1512,7 \\
\hline Cobre (Cu) & 5235,5 & $\begin{array}{c}20334 \\
, 9\end{array}$ & 7382,1 & 46898,1 & 15576,9 & $\begin{array}{c}10028 \\
9\end{array}$ & 2316,2 & 2601,2 & 2174,8 & 2153 \\
\hline $\begin{array}{l}\text { Molibdeno } \\
\text { (Mo) }\end{array}$ & 6,7 & 16,9 & 18,0 & 227,1 & 75,7 & 82,4 & 21,8 & 23,6 & 21,8 & 23,7 \\
\hline Plomo $(\mathrm{Pb})$ & 323,8 & 966,3 & 486,4 & 3154,1 & 765,1 & 842,9 & 144,9 & 174,2 & 165,2 & 125,3 \\
\hline
\end{tabular}

Fuente: ISP (2014) en Hernando (2015), Bolados (2015).

La tabla 5, según la Norma holandesa, da cuenta de que la población está siendo gravemente expuesta a una contaminación aguda y crónica, no solo de arsénico, elemento del que se posee mayor evidencia de sus riesgos y efectos para la salud a nivel mundial, sino también de plomo, que fue la contaminación más percibida por la población y que generó las principales denuncias a finales de la década de los 90'. Sin embargo, nada se ha planteado acerca de los niveles tóxicos de cobre $(\mathrm{Cu})$, zinc $(\mathrm{Zn})$, cadmio $(\mathrm{Cd})$ y de la presencia de contaminación de bario $(\mathrm{Ba})$ y molibdeno (Mo). Toda la ciudad se encuentra contaminada, por una acción sistemática, referida al traslado de material desde los lugares de extracción hacia los lugares de acopio, primero del ferrocarril (en la década de los 90') y en la actualidad por el puerto. Por lo tanto, la contaminación del extractivismo de la GM, que pareciese no afectar, debido a su lejanía con las ciudades, ahora no solo condena a la población por la contaminación del agua, sino también por la contaminación del lugar donde se desarrolla la vida. Los efectos, al parecer, esperan ser medidos en la lógica de Ensayo Clínico; en el que las personas, las familias y comunidades, ni siquiera advierten de su intoxicación y condena.

\section{Discusiones. Necrocapitalismo y biociudadania}

\section{La Necropolítica del Mercado y nuevas formas de Ciudadanía}

Se desea aclarar que el término Necrocapitalismo, acuñado por Banerjee (2008), puede ser definido como "las formas contemporáneas de la acumulación de organización que implican la desposesión y el sometimiento de la vida al poder de la muerte" (p. 1541) y este nace del 
concepto de Necropolítica de Mbembe (2011), "que es la subyugación de la vida a las políticas de la muerte" (p. 75).

Ambos conceptos, destacan el carácter colonialista, tanto del accionar del Estado-Nación, como del Mercado y de la acumulación capitalista. Es por ello necesario plantear y develar que, cuando se trata de los países en vías de desarrollo, las políticas estatales y las políticas del mercado, son muchas veces opuestas a las que operan en países desarrollados; lo que produce y reproduce desigualdad, en la distribución de los daños, riesgos y muertes generadas por la implementación de dichas políticas.

A nivel planetario, es posible reconocer evidencias del accionar conjunto de Necropolítica y Necrocapitalismo, como el caso de Linfen en China y Chittagong en Bangladesh. Järup (2003) plantea que, en la realidad, no existen casos aislados de contaminación, sino una exposición a distintos niveles de esta en el planeta; en este sentido, solo los países desarrollados cuentan con técnicas y normativas más estrictas, para discriminar el nivel de exposición entre inocuidad y nocividad. Lo que nos retrotrae a la hipótesis del colonialismo necropolítico y necrocapitalista, en el que -al parecer- hay áreas del planeta y dentro de los propios Estados-Nación, en donde es factible contaminar y producir la muerte, en pos de mantener otras áreas limpias y protegidas.

Se reflexiona que, en el caso del desarrollismo chileno y de su minería, los productos rápidamente se exportan (desde la minería del salitre en adelante). Sin embargo, no se repara en los costos medioambientales, ni el daño a la población que produce dicho modelo extractivista de materias primas; modelo que ha convertido a la Región de Antofagasta en un Ensayo Clínico a cielo abierto, territorialidad en la que los analistas internacionales, estudian el efecto de la exposición prolongada a metales pesados y arsénico, en las poblaciones que habitan la región.

Otro factor relevante de analizar, tiene relación con el accionar en conjunto del Estado-Nación y el capitalismo en clave neoliberal; dicho amalgamamiento, en el contexto chileno, se da desde el año 1975 a la fecha, configurando un laboratorio neoliberal, con alta participación del empresariado en la toma de decisiones políticas (Huneuss, 2014), (Álvarez, 2015). El análisis de Banerjee (2008) plantea lo siguiente:

En las formas contemporáneas de la acumulación, la corporación es un poderoso actor y en conjunto con los estados nacionales, organismos supranacionales y organismos internacionales, contribuye a una privatización necrocapitalista de soberanía. Para la transformación del colonialismo europeo a un nuevo imperialismo sin colonias, se requiere poder coercitivo y la fuerza bruta, a menudo con la participación de las elites políticas postcoloniales, en las antiguas colonias donde los estados locales surgieron como sitios de poder para la acumulación capitalista (Banerjee, 2008, p. 1549). 
Harvey (2007) plantea que, "de acuerdo con la teoría, el Estado neoliberal debería favorecer unos fuertes derechos de propiedad privada individual, el imperio de la ley y las instituciones del libre mercado y del libre comercio" (p. 71). Empero, el caso chileno tendría presentes las contradicciones y tensiones que plantea el autor -como la formación de monopolios-, sin embargo, el acápite desarrollado en relación a los fallos del mercado es esclarecedor, en torno a los costes vitales; en el caso de Antofagasta, respecto de las vidas humanas contaminadas y desperdiciadas. Según esto:

[Se] eluden asumir la totalidad de los costes imputables a su actividad, eludiendo sus responsabilidades al no permitir que el mercado valore su incidencia mediante el sistema de precios resultante (estas responsabilidades son, en lenguaje técnico, "externalizadas"). El tema clásico para abordar este problema es la contaminación, puesto que los individuos y las compañías eluden los costes vertiendo gratis sus residuos tóxicos en el medio ambiente. Como resultado de su actuación, puede producirse la destrucción o degradación de ecosistemas productivos. La exposición a sustancias peligrosas o a peligros físicos en los centros de trabajo puede afectar a la salud de los seres humanos e incluso reducir la reserva de trabajadores sanos que constituyen la fuerza de trabajo. Aunque los defensores del neoliberalismo admiten la existencia del problema y algunos aceptan la necesidad de una limitada intervención estatal, otros defienden la inacción porque el remedio será casi con toda seguridad peor que la enfermedad (Harvey, 2007, p. 74-75).

Harvey describe la degradación medioambiental, acaecida por la lógica neoliberal (Harvey 2007, p. 179-182); salvo que la problemática analizada, en el presente caso, es de más larga data (desde el año 1930 a la fecha). Por lo tanto, sería correcto decir que, desde la instalación de modelos de desarrollo capitalistas en Chile, la situación de la contaminación de Antofagasta ha pasado inadvertida e invisibilizada, lo que implica asumir que -en este momento neoliberal de la economía chilena- es irrelevante. Lo anteriormente planteado, permite aducir que el Necrocapitalismo, en clave neoliberal, es brutal, ya que acalla la evidencia empírica (Valencia, 2010 , p. 139-172) e ignora las vidas humanas.

El caso de Antofagasta es paradigmático, en tanto las denuncias de la comunidad, en el año 1989, solo son escuchadas 4 años después (en el año 1993), dando paso a la realización del primer estudio. La evidencia producida, permitió dar cuenta de los niveles de contaminación que afectaban a la población (siendo solo descrita la contaminación plúmbica); sin embargo, para develar la dramática situación generada por la contaminación por arsénico, se debe esperar hasta el año 2014, con el estudio realizado por el ISP, aun cuando Chile y la Región de Antofagasta, eran reconocidos e identificados, a nivel mundial, por la Revista Médica Británica The Lancet, como Ensayo Clínico de contaminación a gran escala (Fraser, 2012). 
Por otro lado, este Ensayo Clínico a gran escala, al parecer, según lo plantea Fraser (2012), ocurriría sin la intervención de nadie; en este sentido, habría que oponer la hipótesis de Adriana Petryna (2009), cuyo planteamiento, respecto de los ensayos clínicos, indica que se realizan en lugares donde la regulación es débil y efectivamente es posible la intervención de las multinacionales farmacéuticas sin restricciones. Guardando las proporciones y realizando la analogía necesaria, este Ensayo Clínico, en la Región de Antofagasta, no sería del todo natural, porque, de ser así, esta contaminación no se concentraría en el lugar de acopio de los productos mineros para su exportación; por lo tanto, el argumento de Fraser, de impresión naturalista y objetiva, se despeña ante los hechos empíricos, previamente presentados.

Rabinow (1996) fue el primero en avizorar una transformación de las relaciones entre formas contemporáneas de subjetivación y salud; a partir de sus investigaciones se acuña el término de Biosociabilidad, referente a prácticas colectivas e individuales en torno a la vida, la salud y los derechos. Este planteamiento constituye una reformulación de las perspectivas de acercamiento a la ciudadanía, entendida de forma clásica, desde Thomas Marshall hasta la actualidad.

Por otra parte, Adriana Petryna (2002), Carlos Novas y Nikolas Rose (2004), plantean el término Ciudadanía Biológica o Biociudadanía. Petryna, analizando el caso de Chernóbil (Ucrania), nos devela como los ciudadanos afectados por la explosión del reactor nuclear, exigieron el reconocimiento del derecho a indemnización y reclamaron una redistribución de los recursos políticos, respecto del daño provocado sobre su biología.

El concepto de ciudadanía biológica arroja luz sobre una práctica fundamental de la construcción política en el post socialismo. Análisis recientes de post-socialistas y del mercado de transiciones, han revelado las acciones de estados-nación en relación a la legitimidad de las vidas de las personas. Estas etnografías han trazado el camino de narrativas locales que abordan el colapso del socialismo de Estado y de la coyuntura repentina del capitalismo, la globalización y las nuevas leyes (Petryna, 2002, p. 6) (Traducido para el presente texto).

Novas y Rose (2004), se deslindan de la perspectiva anterior, para dar cuenta de que -desde el siglo XVIII- la ciudadanía ha estado ligada en parte a la biología, características vitales y orgánicas de los individuos, por ello indican lo siguiente:

Los procesos de construcción nacional de los estados europeos y sus colonias, desde a lo menos la mitad del siglo XIX, junto a las ideas y el ejercicio de la ciudadanía, entrañaron modos de conducirse en relación con la salud y la reproducción. Y para la Biopolítica de la primera mitad del siglo XX - sea en su forma eugénica o asistencialista- el cuerpo del ciudadano, el ciudadano individual y el 
cuerpo colectivo del pueblo, la nación y el volk ${ }^{5}$ constituyó un valor fundamental

(Rose 2004, p. 62).

Es esta Biociudadanía, la que se encuentra expuesta a la contaminación en Antofagasta; al riesgo de enfermar y morir, a causa de la exposición continua a metales pesados y arsénico. Sin embargo, es necesaria la acción individual y colectiva, la visibilización de la problemática y la correspondiente interpelación al Estado-Nación chileno y a la industria minera (chilena y extranjera).

Según lo planteado por Folchi (2003), Svampa y Álvarez (2010) y Bowen et al (2012), estamos en presencia de un nuevo actor social total; uno que va en contra de la contaminación y el desastre ambiental, que se produce por el modelo extractivista en América Latina en lo general y en Chile en lo particular. Sin embargo, en el contexto de Antofagasta, este es un movimiento de carácter reciente, que empero aglutina a un sector transversal de la población.

\section{A modo de conclusión}

En primer término, dadas las características y magnitud de la contaminación en la región y de acuerdo con la evidencia disponible, cabe preguntarse ¿por qué no se han realizado acciones frente al envenenamiento y a la mortalidad dosis/dependiente a la que está expuesta la comunidad de Antofagasta y la población de la región en su totalidad? Aun cuando todos los estudios realizados recomiendan, al menos, informar a la población y otorgarles las posibilidades de diagnóstico y tratamiento.

Por otra parte, se constata un accionar negligente de la SEREMI de Antofagasta y la CONAMA, dado que no llevaron a cabo una medición acuciosa de la contaminación, limitándose únicamente a la medición del plomo, dejando de lado otros metales pesados y el arsénico, que fue un hallazgo de las mediciones del ISP, en el año 2014.

En tercer término, se está en desacuerdo con lo descrito por Fraser, en tanto declara que las causas de la contaminación en los cursos de agua, se relacionan con la presencia de volcanes en la zona; siendo que, la ciudad de Antofagasta, presenta mayores concentraciones de contaminantes que toda la región. Por lo tanto, se asume que dicha contaminación no es azarosa, ni producida por causas naturales -como una explicación plausible de dicho eventosino generada directamente por la acción minera y el acopio de material contaminante en el puerto, sin ninguna medida de protección.

En cuarto lugar, cabe preguntarse ¿por qué las autoridades (locales, regionales y nacionales), como así también el empresariado minero, han demorado -más de 14 años- en ejecutar acciones frente al envenenamiento progresivo y la contaminación de la población en la Región de Antofagasta? Análogamente, surge una interrogante complementaria ¿por qué no se han adoptado medidas más estrictas, en lo referente a contaminación por metales pesados y arsénico en la región y el país? responsabilizando a quienes cometan acciones de contaminación.

Se concluye, desde la perspectiva Necropolítica, que la inacción del Estado-Nación chileno, en confluencia con el sector minero presente en la región, da cuenta de una acción necrocapitalista

\footnotetext{
${ }^{5}$ Debe entenderse el Volk como un concepto en la teoría política en clave alemana, que organiza y articula los conceptos de pueblo, nación y población.
} 
concertada. En este sentido, las vidas de las personas, comunidades y poblaciones de la región, son parte del costo vital de la extracción minera; por lo tanto, un costo inevitable, que dado el contexto neoliberal, no sería responsabilidad de un actor particular, si no parte de la propia opacidad de las fallas del mercado (Harvey, 2007).

Por último, es menester evidenciar lo necesario de las nuevas ciudadanías, que confluyan como actores sociales totales, con la posibilidad de generar acciones individuales y colectivas, en pos de una abogacía, control social y accountability transformadores; exigiendo respuestas al Estado-Nación chileno y a las empresas mineras involucradas, configurándose en términos de una Biosociabilidad y Biociudadanía revitalizadora.

\section{Referencias}

Álvarez, R. (2015). Gremios empresariales, política y neoliberalismo. Los casos de Chile y Perú (1986-2010). Santiago de Chile: Lom Ediciones.

Bagchi, S. (2007). Arsenic threat reaching global dimensions. Canadian Medical Association Journal, 177 (11), 1344-1345. Recuperado de: https://doi.org/10.1503/cmaj.071456.

Banerjee, S. B. (2008). Necrocapitalism. Organization Studies, 29 (12), 1541-1563.

Bolados, A. (2015). Bioética para una Antofagasta bajo grave riesgo por efecto de la contaminación. Ponencia en XVI Jornadas de Nacionales de Bioética. Antofagasta. Chile.

Bowen, S., Fábrega, F., Medel, R. (2012). Movimientos Sociales Rurales y Problemática Medioambiental: La Disputa por la Territorialidad. Psicoperspectivas, 11 (1), 204-225. Recuperado de: http://www.scielo.cl/scielo.php?script=sci_arttext\&pid=S071869242012000100010\&Ing=es\&tIng=es. 10.5027/psicoperspectivas-Vol11-Issue1-fulltext187.

Cámara de Diputados. (2016). Informe de la comisión especial investigadora de la actuación de los organismos públicos encargados de la protección de la salud y el medio ambiente en la comuna de antofagasta. Recuperado de: https://www.camara.cl/pdf.aspx?prmID=16907\&prmTIPO=INFORMECOMISION

Cámara de Diputados. (2019). Informe comisión especial investigadora sobre causas de alta contaminación ambiental, especialmente en concón, quintero y puchuncaví, y de responsabilidades en ejecución del plan de descontaminación. Recuperado de: https://www.camara.cl/pdf.aspx?prmID=45601\&prmTIPO=INFORMECOMISION.

Departamento de Estadísticas e Información en Salud (DEIS-MINSAL). (1970-2013). Anuarios de Estadísticas Vitales. Ministerio de Salud. Chile.

Eterovic, R. M. (1998). Comentario 2: Contaminacion, ilegalidad y medidas cautelares en el caso de acopio y transporte de plomo en antofagasta. Revista Chilena de Derecho. Pontificia Universidad Católica de Chile. Recuperado de: https://doi.org/10.2307/41609482.

Folchi, D. M. (2003). La insustentabilidad del boom minero chileno: política y medio ambiente, 1983-2003. Ecología política: cuadernos de debate internacional, (26), 23-49.

Fraser, B. (2012). Cancer cluster in Chile linked to arsenic contamination. The Lancet, 379(9816), 603.

Hartl, G. 2004. WHO issues revised drinking water guidelines to help prevent water related outbreaks and disease. Recuperado de: http:// www.who.int/mediacentre/news/releases/2004/pr67/en/index.html.

Harvey, D. (2007). Breve historia del neoliberalismo. Ediciones Akal.

Hernando, M. (2015). Evidencia de la evolución en la contaminación por metales pesados en la 
ciudad

población

de

Antofagasta.

Estudios

Epidemiológicos $y$

Medioambientales

1993 - 2014. Ponencia en XVI Jornadas de Nacionales de Bioética. Antofagasta. Chile.

Huneeus, C. (2014). La democracia semisoberana: Chile después de Pinochet. Taurus.

Järup, L. (2003). Hazards of heavy metal contamination. British Medical Bulletin, 68 (1), 167182. Recuperado de: https://doi.org/10.1093/bmb/ldg032.

Marshall, G., Ferreccio, C., Yuan, Y., Bates, M. N., Steinmaus, C., Selvin, S., Smith, A. H. (2007). Fifty-Year Study of Lung and Bladder Cancer Mortality in Chile Related to Arsenic in Drinking Water. JNCI Journal of the National Cancer Institute, 99 (12), 920-928. Recuperado de: https://doi.org/10.1093/jnci/djm004.

Mbembe, A. (2011). Necropolítica: seguido de Sobre el gobierno privado indirecto. E. F. Archambault (Ed.). Melusina.

Petryna, A. (2002) Life Exposed: Biological Citizens after Chernobyl. Princeton: Princeton University Press.

Petryna, A. (2009). When experiments travel: clinical trials and the global search for human subjects. United States: Princeton University Press.

Rabinow, P. (1996). Essays on the Anthropology of Reason. United States: Princeton University Press.

Ravenscroft, P. (2007). Predicting the global distribution of natural arsenic contamination of groundwater. In Symposium on arsenic: the geography of a global problem. London: Royal Geographical Society.

Rose, N., \& Novas, C. (2004). Biological citizenship (pp. 439-463). Blackwell Publishing.

Rose, N. (2012). Políticas de la vida: biomedicina, poder y subjetividad en el siglo XXI. UNIPE: Editorial Universitaria.

Sepúlveda, V., Vega, J. \& Delgado, I. (2000). Exposición severa a plomo ambiental en una población infantil de Antofagasta, Chile. Revista Médica de Chile, 128 (2), 221-232. Recuperado de: https://doi.org/10.4067/S0034-98872000000200014.

Smith, A. H., Lopipero, P. A., Bates, M. N. \& Steinmaus, C. M. (2002). Arsenic epidemiology and drinking water standards. Science, 296 (5576), 2145-2146.

Smith, A. H., \& Steinmaus, C. M. (2009). Health effects of arsenic and chromium in drinking water: recent human findings. Annual review of public health, 30, 107.

Smith, A. H., Marshall, G., Yuan, Y., Liaw, J., Ferreccio, C., \& Steinmaus, C. (2011). Evidence From Chile That Arsenic in Drinking Water May Increase Mortality From Pulmonary Tuberculosis. American Journal of Epidemiology, 173 (4), 414-420. Recuperado de: https://doi.org/10.1093/aje/kwq383.

Steinmaus, C. M., Ferreccio, C., Romo, J. A., Yuan, Y., Cortes, S., Marshall, G. \& Smith, A. H. (2013). Drinking water arsenic in northern Chile: high cancer risks 40 years after exposure cessation. Cancer Epidemiology Biomarkers \& Prevention, 22 (4), 623-630.

Svampa, M. y Álvarez, M. S. (2010). Modelo minero, resistencias sociales y estilos de desarrollo: los marcos de la discusión en la Argentina. Revista Ecuador Debate, 79, 106125.

Valencia, S. (2010). Capitalismo gore. Melusina.

World Health Organization. (2004). Guidelines for drinking-water quality: recommendations (Vol. 1). World Health Organization.

\section{Referencias electrónicas}

https://www.camara.cl/prensa/noticias_detalle.aspx?prmid=125715

http://www.iarc.fr. 